PROCEEDINGS OF THE AMERICAN MATHEMATICAL SOCIETY

Volume 126, Number 3, March 1998, Pages 925-931

S 0002-9939(98)04225-7

\title{
FOLIATIONS OF SOME 3-MANIFOLDS WHICH FIBER OVER THE CIRCLE
}

\author{
D. COOPER AND D. D. LONG
}

(Communicated by Ronald A. Fintushel)

\begin{abstract}
We show that a hyperbolic punctured torus bundle admits a foliation by lines which is covered by a product foliation. Thus its fundamental group acts freely on the plane.
\end{abstract}

\section{INTRODUCTION}

This paper discusses one dimensional foliations of closed three-manifolds. Every closed three-manifold admits a one dimensional foliation; for example the threesphere admits a foliation by round circles (Hopf) and by smooth lines [4]. Epstein, [3], showed that every foliation by circles is a Seifert fibration, and this class of manifolds has been extensively studied. A manifold which fibers over the circle admits a one dimensional foliation such that each leaf maps monotonely under the map to the circle defining the fibration. If a closed three-manifold admits one of the eight geometric structures described by Thurston, [5], then it is either Seifert fibered, a torus bundle over the circle, or hyperbolic. It has been conjectured that every hyperbolic three-manifold is finitely covered by a three-manifold which fibers over the circle. A foliation of $M$ induces a foliation on any cover of $M$ by pull-back. In the case that $M$ fibers over the circle, or is Seifert fibered, either the universal cover is the three-sphere or is foliated as a product. This suggests looking at a common generalization of these types of foliation.

Definition 1.1. A one-dimensional foliation of a three-manifold $M$ is called product covered if the foliation is covered by a product foliation of the universal cover of $M$.

When does a three-manifold $M$ admit a product covered one dimensional foliation? One reason that such foliations are of interest is:

Theorem 1.2. Suppose that a three-manifold $M$ admits a product covered foliation with no closed leaves. Then either $M$ is a line bundle over $\mathbb{R} P^{2}$ or $\pi_{1} M$ acts freely on the plane.

Proof. By hypothesis the universal cover of $M$ is a product $F \times \mathbb{R}$ with $F$ a surface. Now $F$ is simply connected and the action by covering transformations on $\tilde{M}$

Received by the editors June 16, 1996 and, in revised form, September 4, 1996.

1991 Mathematics Subject Classification. Primary 57M50, 57M60, 57R30.

Key words and phrases. 3-manifold, foliation, product-covered, surface-bundle.

Both authors was supported in part by NSF.

(C)1998 American Mathematical Society 
induces an action on $F$. If some non-trivial element $\alpha$ of $\pi_{1} M$ fixes a point $x$ of $F$ then $\alpha$ maps the leaf $x \times \mathbb{R}$ to itself freely and so the image of this leaf in $M$ is a circle, a contradiction. Thus $\pi_{1} M$ acts freely on $F$. If $F$ is a sphere then every orientation preserving homeomorphism of $F$ has a fixed point; hence $\pi_{1} M$ has at most two elements. Thus if $F$ is a sphere and $\pi_{1} M$ is non-trivial then $M$ is a line bundle over $\mathbb{R} P^{2}$. Otherwise $\pi_{1} F$ acts freely on the interior of $F$ which is a plane as asserted.

This suggests further questions: which three-manifolds have fundamental groups that act faithfully on the plane?, which act freely on the plane? In this paper we construct some product covered foliations without closed leaves for bundles over the circle where the fiber is a torus or punctured torus. In the case of a punctured torus the proof requires that the monodromy is pseudo-Anosov. In particular the Figure8 knot complement has such a foliation; thus its fundamental group acts freely on the plane. Since this group contains the fundamental group of some closed surface of genus at least 2, we obtain a rather surprising corollary:

Theorem 1.3. There is a free action of the fundamental group of some closed surface of genus $g \geq 2$ on the plane which is not properly discontinuous.

If $\Gamma$ is a non-elementary subgroup of the isometries of the hyperbolic plane $\mathbb{H}^{2}$ and $\Gamma$ does not act properly discontinuously on the hyperbolic plane then $\Gamma$ contains an elliptic (for example see [1] Theorem (8.3.1)). Thus one cannot obtain an example of the action provided by the theorem as a subgroup of $P S L_{2} \mathbb{R}$.

\section{Torus BundLES}

Let us start with a torus bundle:

$$
T^{2} \longrightarrow N \longrightarrow S^{1}
$$

with monodromy $\phi$. We first construct a nowhere zero vector field called a horizontal vector field on $N$ as follows. Regard $N$ as $T^{2} \times[0,1]$ with the boundary components identified via $\phi$. Choose, for each $t \in[0,1]$, a unit vector $h_{t}$ tangent to $T^{2}$ such that $h_{t}$ varies continuously with $t$ and such that $\phi$ identifies $h_{0}$ with $h_{1}$. This may be done by monotonically increasing the angle, $\theta_{t}$, that $h_{t}$ makes with some fixed direction in $T^{2}$. We may choose coordinates so that $0<\theta_{0}<\theta_{1}<2 \pi$. In addition we may choose $\theta_{0}$ so that the foliation of $T^{2}$ by leaves parallel to $\theta_{0}$ is a foliation all of whose leaves are non-compact. Each flow line of $h$ is contained in some horizontal torus $T^{2} \times t$ and may be compact or non-compact. However the flow lines at the 0 and 1 levels are non-compact.

We will now tilt the flow lines in the levels in $(0,1)$ by adding an upwards component to the horizontal vector field. The result will be a new vector field with all integral curves non-compact. Now we construct another vector field $v$ on $N$ called a vertical vector field by taking a smooth function $f$ on $[0,1]$ which is zero at the endpoints and positive everywhere else. Then $v_{t}=f(t) . e$ where $e$ is a unit vector in the $[0,1]$ direction on $T^{2} \times[0,1]$. Thus the vector field $w=h+v$ on $N$ determines a foliation $\mathcal{F}$ of $N$ by lines.

Choose a vector on $T^{2}$ making an angle which is not in the interval $\left[\theta_{0}, \theta_{1}\right]$ and such that an integral curve on $T^{2}$ in the direction of this vector is a simple closed curve $C$. Then $C$ is transverse to every leaf of $h_{t}$ for each $t$. Let $\tilde{N}$ be the infinite cyclic cover $T^{2} \times \mathbb{R}$ of $N$ given by unwrapping the $S^{1}$ direction. Then $A=C \times \mathbb{R}$ 
is an annulus in $\tilde{N}$. Let $\tilde{A}$ be a pre-image of $A$ in the universal cover on $N$. It is easy to see that $\tilde{A}$ meets every flow line once transversally. This proves that $\mathcal{F}$ is product covered, so we have shown:

Theorem 2.1. Every torus bundle over the circle admits a foliation by lines which is product-covered.

\section{BLOWING UP LEAVES}

Let $S$ be a countable subset of the line $L$ and

$$
\pi: L^{+} \longrightarrow L
$$

a continuous map from the line $L^{+}$onto the line $L$ such that the pre-image of a point in $S$ is an interval of positive length and the pre-image of every point not in $S$ is a single point. We say that $L^{+}$is the result of blowing up the set $S$.

Suppose now that $G$ is a group of homeomorphisms acting on $L$ and that $S$ is invariant under $G$. We wish to lift this action to an action of $G$ on $L^{+}$which is semi-conjugate to the original action, in other words so that the following diagram commutes:

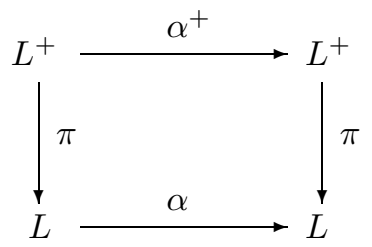

To do this we describe a canonical way to lift a homeomorphism $\alpha$ of $L$ which preserves $S$ to a homeomorphism $\alpha^{+}$of $L^{+}$covering $\alpha$, i.e. so that $\pi \alpha^{+}=\alpha \pi$. Choose a metric on $L^{+}$equivalent to the usual metric on $\mathbb{R}^{1}$. The condition on $\alpha^{+}$ uniquely determines the image of a point $x$ of $L^{+}$in the case that $\pi x$ is not in $S$, namely

$$
\alpha^{+} x=\pi^{-1} \alpha \pi(x) .
$$

In the case that $\pi x$ is in $S$ then $I=\pi^{-1} \pi(x)$ is an interval, and since $S$ is preserved by $\alpha$ we have that $\pi^{-1} \alpha \pi(x)$ is an interval $J$ of positive length. The metric on $L^{+}$ determines a unique linear map of $I$ onto $J$. If $\alpha$ preserves the orientation of $L$, then this linear map is chosen to be increasing; otherwise it is chosen to be decreasing.

Now it is clear that $\alpha^{+}$is monotonic and surjective thus a homeomorphism. Furthermore it is clear that

$$
(\alpha \circ \beta)^{+}=\alpha^{+} \circ \beta^{+}
$$

since the composition of linear maps is linear. Thus we have:

Theorem 3.1. Suppose that $S$ is a countable subset of a line $L$ and that a group $G$ acts by homeomorpisms on $L$ and preserves $S$. Then there are a blowup $\pi: L^{+} \longrightarrow L$ of $L$ and an action of $G$ on $L^{+}$by homeomorphisms which is semi-conjugate via $\pi$ to the action on $L$. 


\section{Hyperbolic PUNCTURED TORUS BUNDLES}

Let $M$ be a three-manifold which fibers over the circle with fiber a punctured torus $F$, and pseudo-Anosov monodromy, $\phi$; thus

$$
F \longrightarrow M \longrightarrow S^{1}
$$

Let $N$ be the associated torus bundle with Anosov monodromy $\phi$. Thus there is a point on $T^{2}$ fixed by the monodromy so that if $C$ is the circle in $N$ which is the suspension of this point, then $M=N-C$. We will construct a product covered foliation on $N$ with $C$ as the only closed leaf. Then the restriction of this foliation to $M$ is a product covered foliation with no closed leaf. The reason is that since the foliation on $N$ is product covered, there is a plane, $P$, in $\tilde{N}$ which meets every leaf once transversally. Let

$$
p: \tilde{N} \longrightarrow N
$$

be the universal cover. Then $S=P \cap p^{-1}(C)$ is a closed subset of $P$ and thus the complement $P^{-}=P \cap p^{-1}(N-C)$ of $P$ is a surface which is the leaf space of the foliation restricted to $p^{-1}(N-C)$. Since this is a product it follows that the foliation of $N-C$ is product covered.

To construct the foliation $\mathcal{F}$ on $N$ we start with a foliation $\mathcal{F}_{1}$ on $N$ whose leaves are horizontal lines on the tori $T^{2} \times t$ which are parallel to an eigenvector $u$ of the monodromy $\phi$. The universal cover $\tilde{N}$ may be identified with $\mathbb{R}^{3}$ so that the planes $z=$ constant project linearly to the horizontal tori $T^{2} \times t$ in $N$ and so that lines parallel to the $x$ and $y$ axes project to straight lines parallel to the eigenvectors of $\phi$. This is done so that the vector $u$ lies on the $x$-axis. Thus the foliation $\mathcal{F}_{1}$ is covered by a foliation $\tilde{\mathcal{F}}_{1}$ of $\mathbb{R}^{3}$ by lines parallel to the $x$-axis. Now the action of $\pi_{1} N$ on $\mathbb{R}^{3}$ factors into actions on each $\mathbb{R}^{1}$ factor. To see this, the fundamental group of the horizontal torus $T^{2} \times 0$ acts by translations in the $x y$ plane. The element of $\pi_{1} N$ represented by $C$ acts by a diagonal linear map on the $x y$ plane composed with a translation in the $z$-direction. These elements generate $\pi_{1} N$ which proves the claim.

Next we blow up the annulus $A$ consisting of leaves meeting $C$. This is done as follows. The preimage $p^{-1}(A)$ consists of a dense set of planes each with equation $y=$ constant. Let $S$ be the countable subset of the $y$-axis consisting of points on these planes. This subset is preserved by the action of $\pi_{1} N$. Thus we may blow up $S$ equivariantly with respect to this action. Using the product structure, we may thus blow up this set of planes in $\mathbb{R}^{3}$ equivariantly. The quotient by the group action is a three-manifold $N^{+}$equipped with a quotient map

$$
\pi: N^{+} \longrightarrow N
$$

which is homotopic to a homeomorphism. Also $N^{+}$is foliated by the projection of the planes $y=$ constant and this 2-dimensional foliation is the result of blowing up $A$ in the 2-dimensional foliation of $N$ coming from the planes $y=$ constant.

The annulus $A$ has a foliation, $\mathcal{C}$, by circles which each meet each torus $T^{2} \times t$ once; such a foliation is called vertical. We may arrange that $C$ is one of the leaves of $\mathcal{C}$.

We now describe the foliation $\mathcal{F}$ on $N^{+}$(which is homeomorphic to $N$ ). The quotient map is a bijection between $\left(N^{+}-\pi^{-1} A\right)$ and $N-A$ and the leaves of $\mathcal{F}$ in $\left(N^{+}-\pi^{-1} A\right)$ are the preimages of the leaves of $\mathcal{F}_{1}$. The foliation on the boundary 
$\mathrm{f}(\mathrm{x})$

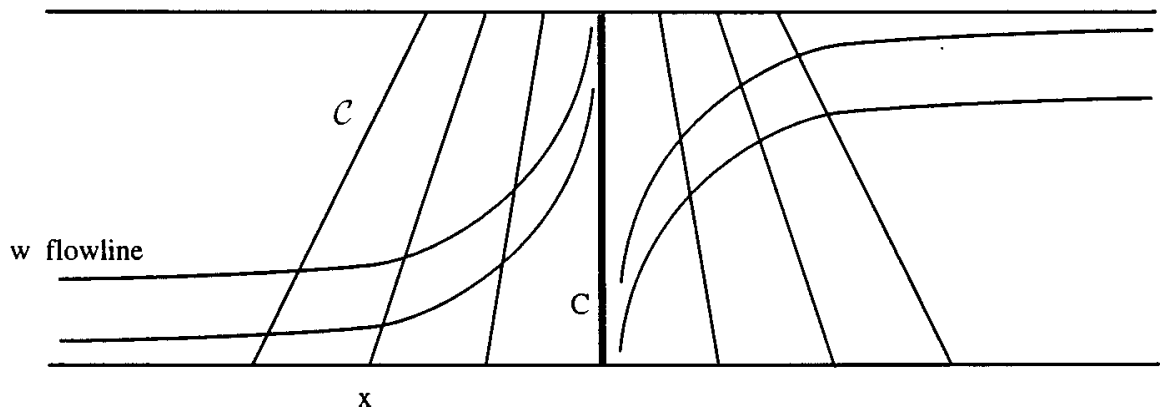

Figure 1. The middle annulus

$A \times\{ \pm 1\}$ is by horizontal lines, and the blown up annulus $\pi^{-1} A=A \times[-1,1] \subset N^{+}$ is foliated in a way that extends this foliation on the boundary.

We now describe $x, y, z$ coordinates on $\tilde{N}^{+}$. The blowing-up procedure used to obtain $N^{+}$from $N$ is covered by a $\pi_{1} N$-equivariant blow-up of $\mathbb{R}^{3}$. This involves blowing up a countable set of points on the $y$-axis and extending product-wise over $\mathbb{R}^{3}$. We impose coordinates on $\tilde{N}^{+}$obtained by this blow-up procedure. Thus the quotient map $\tilde{\pi}: \tilde{N}^{+} \longrightarrow \tilde{N}$ covering $\pi$ preserves the $x$ and $z$ coordinates.

These coordinates on $\tilde{N}^{+}$together with the covering space projection

$$
p^{+}: \tilde{N}^{+} \longrightarrow N^{+}
$$

give an identification of $A \equiv A \times 0$ with $\mathbb{R} \times 0 \times I /(x, 0,0) \equiv(\lambda x, 0,1)$. We also use an identification of $A \times[-1,1]$ with a quotient of $\mathbb{R} \times[-1,1] \times I$. The top is identified with the bottom by the identification $(x, f(y), 0) \equiv(\lambda x, y, 1)$ where the $x, y, z$ coordinates are as described above. The map $f$ is a diffeomorphism of $[-1,1]$ such that $-1,0,1$ are the only fixed points and for $0<|y|<1$ we have $0<|f(y)|<|y|$. Let $u$ be the unit vector field on $\mathbb{R} \times[-1,1] \times I$ in the horizontal $\mathbb{R}$ direction (i.e. parallel to the $x$-axis). Let $v$ be the unit vector field in the vertical $I$ direction (i.e. parallel to the $z$-axis). Let $g$ be a smooth non-negative function

$$
g: A \times[-1,1] \longrightarrow[0,1]
$$

such that $g^{-1}(1)=C \subset A \times 0$ and $g^{-1}(0)=A \times\{ \pm 1\}$. Consider the vector field on $A \times[-1,1]$

$$
w=g \cdot v+(1-g) \cdot u .
$$

The only closed flow lines of $w$ are in $A \times 0$. This is because for the other flow lines, the $x$ coordinate along a flow line increases until the flow line crosses the $z=1$ level, at which point it moves inwards towards $A \times 0$ (i.e. $|y|$ decreases). Furthermore, we may choose $g$ such that $w$ is transverse, on $A \times 0$, to $\mathcal{C}$ everywhere except $C$. This is done by making $g \rightarrow 1$ sufficiently slow near $C$; thus $w$ is always more nearly horizontal than $\mathcal{C}$. See Figure 1.

To prove that $\mathcal{F}$ is product covered consider the plane, $R$, with equation $x+z=0$ in $\mathbb{R}^{3}$. Then $\mathcal{F}$ is covered by a foliation, $\tilde{\mathcal{F}}$, of $\mathbb{R}^{3}$ such that the image under the blow-down map, $\pi$, of every leaf of $\tilde{\mathcal{F}}$ maps to a line in $\mathbb{R}^{3}$ with monotonic increasing $x$ and $z$ coordinates and such that $x+z$ maps the leaf onto $\mathbb{R}$. Thus the image leaf 
meets $R$ once transversally. The process of blowing up a countable subset of the $y$ axis, and extending this blow-up product-wise to $\mathbb{R}^{3}$ results in the plane $x+z=0$ being blown up to give a new plane with equation $x+z=0$ since only the $y$ coordinate is affected by the blow-up. Thus the pre-image of $R$ under blow up is $\pi^{-1} R$ which is a plane in $\mathbb{R}^{3}$. Hence the pre-image of $R$ is a plane which meets every leaf of $\tilde{\mathcal{F}}$ once transversally, and so $\tilde{\mathcal{F}}$ is a product. This establishes the following theorem. Does it generalize to all hyperbolic three-manifolds which fiber over the circle?

Theorem 4.1. Every hyperbolic three-manifold which is a punctured torus bundle over the circle admits a one-dimensional foliation without closed leaves and which is product-covered.

Let $M$ be the figure- 8 knot complement. Now it is known [2] that there is a map of a closed surface $R$ of some genus $g \geq 2$ into $M$ and a finite cover $N$ of $M$ such that $R$ lifts to an embedded incompressible surface, $S$, in $N$. The free action of $\pi_{1} N$ on the leaf space $\mathbb{R}^{2}$ of the foliation of $\tilde{N}$ restricts to a free action of $\pi_{1} S$ on $\mathbb{R}^{2}$. We claim that this action is not properly discontinuous.

Otherwise the quotient $\mathbb{R}^{2} / \pi_{1} S$ is a closed surface. Thus $\tilde{N} / \pi_{1} S$ is foliated as a product $F \times \mathbb{R}$ with $F \cong S$. Let $S_{1}$ be the lift of $S$ to this covering. Then $S_{1}$ is homotopic to $F$. Therefore $S_{1}$ meets every flowline of $F \times \mathbb{R}$ algebraically once. In particular, the intersection of $S_{1}$ with each flowline is compact. Hence $\tilde{S}_{1}$ meets every flow line in $\tilde{N}$ algebraically once. This property is preserved by the covering transformations, $\pi_{1} N$. Thus every component of $\pi^{-1} S$ meets every flow line. Let

$$
\pi: \tilde{N} \longrightarrow N
$$

be the universal cover. Since $S$ is embedded in $N$, the subsurface $\pi^{-1} S$ consists of a set of disjoint copies of the universal cover of $S$. Choose a leaf $\lambda$ of the foliation of $\tilde{N}$. Each component of $\pi^{-1} S$ meets $\lambda$ algebraically once. Thus there is an upper-most intersection of each component of $\pi^{-1} S$ with $\lambda$. Let $\max (\tilde{S} \cap \lambda)$ be this upper-most intersection, and let $\mathcal{S}_{\lambda}$ be the subset of $\lambda$ consisting of, for each component $\tilde{S}$, the point $\max (\tilde{S} \cap \lambda)$. The linear ordering of points on $\lambda$ defines an ordering on $\mathcal{S}_{\lambda}$ and thus on the set of components of $\pi^{-1} S$. We claim that this ordering is preserved by the action of $\pi_{1} N$. The foliation of $\tilde{N}$ has an orientation which is preserved by the action of $\pi_{1} N$. Now $\tilde{S}_{1}$ is a properly embedded topological plane which separates $\tilde{N}$ into complementary components $A$ and $B$. The notation is chosen so that as you move along every flow line in the direction given by the orientation, you are eventually always in $A$. If $\tilde{S}_{2}$ is another component of $\pi^{-1} S$ contained in $B$ it is clear that given a point $x$ on $\tilde{S}_{2}$ moving up the flow line through $x$ one gets to some point $y$ on $\tilde{S}_{1}$. Thus every choice of flow line yields the same ordering on the set of components of $\pi^{-1} S$. Thus the ordering is preserved by the action of $\pi_{1} N$, proving the claim.

Since $N$ is compact and $\pi$ is an infinite sheeted regular covering it follows that $\pi^{-1} S$ has infinitely many components. It is also clear $\mathcal{S}_{\lambda}$ is discrete. This ordering gives $\lambda$ the structure of a simplicial tree $\Gamma$ on which $\pi_{1} N$ acts. The vertices of $\Gamma$ are the midpoints of components of $\lambda-\mathcal{S}_{\lambda}$ with edges between adjacent components. This tree is isomorphic to the tree obtained from $\tilde{N}$ by taking components of $\tilde{N}-\pi^{-1} S$ as vertices and connecting two vertices by an edge if the closure of the corresponding complementary components intersect. Then $\pi_{1} N$ acts on both trees, and the isomorphism is equivariant. The quotient $\Gamma / \pi_{1} N$ is a circle. We claim that 
this implies that $S$ is a fiber of a fibration of $N$ over the circle. But this is absurd, since $S$ is closed and $N$ is not.

It remains to prove the claim. The tree $\Gamma$ is isomorphic to the tree obtained from $\mathbb{R}$ by using $\mathbb{Z}$ as the vertex set. The action of $\pi_{1} N$ on $\mathbb{R}$ preserves orientation and therefore gives an epimorphism

$$
\theta: \pi_{1} N \longrightarrow \mathbb{Z}
$$

The kernel of $\theta$ is the stabilizer of a vertex and is therefore a conjugate of $\pi_{1} S$. By a theorem of Stallings, [6], it follows that $N$ fibers over the circle with $S$ as a fiber.

Theorem 4.2. There is a free action of the fundamental group of a closed surface of some genus $g \geq 2$ on the plane which is not properly discontinuous.

Observe that trying to arrange that a non-properly discontinuous action is free seems to be delicate, since one might think that non-free actions are dense among such actions. Are they? We thank Sergio Fenley for pointing out an error in an earlier version of the argument that the above action is not properly discontinuous. This leads to the question: if a three-manifold $M$ has a one-dimensional foliation and there is a covering of $M$ so that the induced foliation is the product foliation on $F \times \mathbb{R}$ for some closed surface $F$, then is $F$ a virtual fiber? The above argument shows that if there is a finite cover of $M$ in which some pre-image of the projection of $F$ into $M$ is embedded, then the answer is yes.

\section{REFERENCES}

[1] A.F. Beardon The Geometry of Discrete Groups. Graduate Texts in Math. 91 (1983), Springer-Verlag. MR 85d:22026

[2] D. Cooper, D.D. Long \& A.W. Reid Essential closed surfaces in bounded 3-manifolds. J. Amer. Math. Soc. 10 (1997), no. 3, 553-563.

[3] D.B.A. Epstein Periodic flows on three-dimensional manifolds. Ann. of Math. 95(1972), 6682. MR 44:5981

[4] K. Kuperberg A smooth counterexample to the Seifert conjecture. Ann. of Math. 140 (1994), no. 3, 723-732. MR 95g:57040

[5] G.P. Scott The Geometries of 3-Manifolds. Bull. London Math. Soc. 15(1983), 401-487 MR 84m:57009

[6] J. Stallings On Fibering certain 3-manifolds. Topology of 3-manifolds. 95-100, Prentice Hall (1962) MR 28:1600

Department of Mathematics, University of California, Santa Barbara, California 93106

E-mail address: cooper@math.ucsb.edu

E-mail address: long@math.ucsb.edu 